PROCEEDINGS OF THE

AMERICAN MATHEMATICAL SOCIETY

Volume 132, Number 5, Pages 1463-1471

S 0002-9939(03)07255-1

Article electronically published on November 26, 2003

\title{
UNITARY OPERATORS PRESERVING WAVELETS
}

\author{
ZIEMOWIT RZESZOTNIK AND XIAOFEI ZHANG \\ (Communicated by David R. Larson)
}

\begin{abstract}
We characterize a special class of unitary operators that preserve orthonormal wavelets. In the process we also prove that symmetric wavelet sets cover the real line.
\end{abstract}

\section{INTRODUCTION}

Following Larson and Weiss we are interested in general properties of the set $\widehat{\mathcal{W}}$, that is, the set of Fourier transforms of wavelets. $\widehat{\mathcal{W}}$ is a subset of the sphere with radius $\sqrt{2 \pi}$ in $L^{2}(\mathbb{R}, d \xi)$ and the sphere with radius $\sqrt{2 \log 2}$ in $L^{2}\left(\mathbb{R}, \frac{d \xi}{|\xi|}\right)$. The set $\widehat{\mathcal{W}}$ is characterized by two equations on one sphere (see HKLS, [HW] and by an equivalent couple of equations on the other sphere (see $[\mathbb{B},[\mathbb{R}]$ ). The set of all linear combinations of functions from $\widehat{\mathcal{W}}$ is dense in $L^{2}(\mathbb{R}, d \xi)$ and $L^{2}\left(\mathbb{R}, \frac{d \xi}{\mid \xi}\right)$, and $\widehat{\mathcal{W}}$ itself is closed under the norm $\|\cdot\|_{L^{2}(\mathbb{R}, d \xi)}+\|\cdot\|_{L^{2}\left(\mathbb{R}, \frac{d \xi}{\xi \xi}\right)}$ (see [GS]). Whether $\widehat{\mathcal{W}}$ is a connected set is still an open question.

In this paper, we investigate operators $T$ that are unitary in both norms $\|\cdot\|_{L^{2}(\mathbb{R}, d \xi)},\|\cdot\|_{L^{2}\left(\mathbb{R}, \frac{d \xi}{|\xi|}\right)}$ and preserve the set $\widehat{\mathcal{W}}$ by satisfying the inclusion relation $T(\widehat{\mathcal{W}}) \subset \widehat{\mathcal{W}}$. This is related to [WUTAM] where operators preserving $\widehat{\mathcal{W}}$ given by $T f=\nu f, \nu$ complex-valued, were characterized. In this characterization $\nu$ is a unimodular function such that $\nu(2 \xi) \overline{\nu(\xi)}$ is $2 \pi$-periodic and is called a wavelet multiplier. The only other known continuous linear transformation that preserves $\widehat{\mathcal{W}}$ is the reflection $f(\xi) \rightarrow f(-\xi)$. As pointed out by Larson there are probably no other operators that are unitary in $L^{2}(\mathbb{R}, d \xi)$ and preserve $\widehat{\mathcal{W}}$ (see $[\mathrm{L}]$ ). The question concerning whether these are the only continuous linear transformations preserving $\widehat{\mathcal{W}}$ was later brought up by Weiss [W].

The additional condition we have on the continuous linear operator $T$ in this paper, i.e., $T$ is unitary in the $\|\cdot\|_{L^{2}\left(\mathbb{R}, \frac{d \xi}{\xi}\right)}$ norm as well, implies that such an operator $T$ must be of an explicit form that we can work with. Using symmetric wavelet sets we prove that $T$ is given as a multiplication by $\nu$ and a reflection restricted to a subset $A$ of $\mathbf{R}$. Then an additional use of asymmetric wavelet sets allows us to conclude that $A$ must be either $\emptyset$ or $\mathbf{R}$. That $\nu$ has to be a wavelet multiplier follows from [WUTAM].

Received by the editors September 30, 2002 and, in revised form, January 12, 2003.

2000 Mathematics Subject Classification. Primary 42C15.

Key words and phrases. Orthonormal wavelets, wavelet sets.

(C)2003 American Mathematical Society 
In the final part of our paper we discuss possibilities of avoiding both of these unitarity restrictions imposed on $T$.

\section{Preliminaries}

A function $\psi$ in $L^{2}(\mathbb{R}, d \xi)$ is called a wavelet if the system

$$
\left\{\psi_{j, k}=2^{\frac{j}{2}} \psi\left(2^{j} \cdot-k\right)\right\}_{j, k \in \mathbf{Z}}
$$

forms an orthonormal basis of $L^{2}(\mathbb{R}, d \xi)$. A wavelet set is a subset $W$ of $\mathbf{R}$, such that its characteristic function $\chi_{W}$ is a Fourier transform of a wavelet (we define the Fourier transform by $\left.\hat{f}(\xi)=\int e^{i x \xi} f(x) d x\right)$. Wavelet sets are characterized by two simple equations:

$$
\begin{gathered}
\sum_{k \in \mathbf{Z}} \chi_{W}(\xi+2 k \pi)=1, \\
\sum_{j \in \mathbf{Z}} \chi_{W}\left(2^{j} \xi\right)=1 .
\end{gathered}
$$

Moreover, if $\psi$ satisfies $|\hat{\psi}|=\chi_{W}$, where $W$ is a wavelet set, then $\psi$ is a wavelet.

The following two lemmas are needed for us to construct wavelet sets later. See $[\mathrm{S}]$ for the proof of the first one and $[\mathrm{Z}$ for the proof of the second one.

Lemma 2.1. Let $E$ be a measurable subset of $\mathbb{R}$ such that

(i) $2^{k} E \cap 2^{l} E=\emptyset$ whenever $k \neq l$, for $k, l \in \mathbb{Z}$,

(ii) $(E+2 \pi k) \cap(E+2 \pi l)=\emptyset$ whenever $k \neq l$, for $k, l \in \mathbb{Z}$,

(iii) the multiplicative measure of $E$ (i.e. $\frac{d \xi}{|\xi|}$ ) is less than $2 \log 2$,

(iv) there is a neighborhood $U$ of 0 such that $(U+2 k \pi) \cap E=\emptyset$ for all $k \in \mathbb{Z}$. Then, there is a wavelet set $W$ such that $E \subset W$.

Lemma 2.2. Let $A, B \subset \mathbf{R}$ be measurable sets with finite positive measure. If there exist $n_{1}, n_{2}, k_{1}, k_{2} \in \mathbf{Z}$ such that

(i) $2^{k_{1}} B \subset A+2 n_{1} \pi$,

(ii) $A+2 n_{2} \pi \subset 2^{k_{2}} B$,

(iii) $\left(A+2 n_{2} \pi\right) \cap 2^{k_{1}} B=\emptyset$,

then there exists a measurable set $F \subset\left(A+2 n_{2} \pi\right) \cup 2^{k_{1}} B$ such that $F$ is both $2 \pi$-translation congruent to $A$ and 2 -dilation congruent to $B$.

To apply the above lemma we shall assume that $A$ is bounded and $B$ is contained in $[\theta, 2 \theta)$ for some positive $\theta$. Observe that if $A+2 n_{1} \pi$ contains $(0, \epsilon)$ for some $\epsilon>0$, then the first containment relation can be achieved by taking $k_{1}$ such that $2^{k_{1}} B \subset\left(\frac{\epsilon}{4}, \epsilon\right)$. Moreover, if $B$ has nonempty interior, then the second containment relation can be achieved by taking an appropriately large $k_{2}$ and $n_{2}$ such that $A+2 n_{2} \pi \subset[\epsilon, \infty)$, which also assures condition (iii).

We use Lemma 2.2 to obtain our first result.

Proposition 2.3. Symmetric wavelet sets cover $\mathbf{R} \backslash\{0\}$.

Proof. Observe that if $E$ is one of the sets $[0, \pi),\left[\frac{\pi}{4}, \frac{\pi}{2}\right) \cup\left[\pi, \frac{3}{2} \pi\right) \cup\left[\frac{7}{4} \pi, 2 \pi\right)$ or $\left[\frac{\pi}{2}, \pi\right) \cup\left[\frac{3}{2} \pi, 2 \pi\right)$, then $-E \cup E$ is $2 \pi$-translation congruent to $[0,2 \pi)$. Therefore, if a measurable set $G \subset \mathbf{R}^{+}$is both $2 \pi$-translation congruent to $E$ and 2-dilation congruent to a 2-dilation generator of a measurable partition of $\mathbf{R}^{+}$, then $-G \subset \mathbf{R}^{-}$ is both $2 \pi$-translation congruent to $-E$ and 2-dilation congruent to a 2-dilation 
generator of a measurable partition of $\mathbf{R}^{-}$. Thus, $-G \cup G$ would be a symmetric wavelet set, and we can concentrate on constructing such sets $G$ that will cover $\mathbf{R}^{+}$.

We consider three cases.

1. Cover $(0, \pi)=\bigcup_{k \in \mathbf{N} \cup\{0\}}\left[\frac{\pi}{2^{k+1}}, \frac{\pi}{2^{k}}\right)$.

Fix $k \in \mathbf{N} \cup\{0\}$. Let $G_{k, 0}=\left[\frac{\pi}{2^{k+1}}, \frac{3 \pi}{2^{k+2}}\right) \cup F_{k, 0}$, where $F_{k, 0}$ is both $2 \pi$-translation congruent to $[0, \pi) \backslash\left[\frac{\pi}{2^{k+1}}, \frac{3 \pi}{2^{k+2}}\right)$ and 2-dilation congruent to $\left[\frac{3 \pi}{2^{k+2}}, \frac{\pi}{2^{k}}\right)$. Let $G_{k, 1}=$ $\left[\frac{3 \pi}{2^{k+2}}, \frac{\pi}{2^{k}}\right) \cup F_{k, 1}$, where $F_{k, 1}$ is both $2 \pi$-translation congruent to $[0, \pi) \backslash\left[\frac{3 \pi}{2^{k+2}}, \frac{\pi}{2^{k}}\right)$ and 2-dilation congruent to $\left[\frac{\pi}{2^{k+1}}, \frac{3 \pi}{2^{k+2}}\right)$. The sets $F_{k, 0}$ and $F_{k, 1}$ exist by Lemma 2.2 and the comments following it. Since each of the sets $G_{k, 0}$ and $G_{k, 1}$ is $2 \pi$ translation congruent to $[0, \pi)$ and 2-dilation congruent to $\left[\frac{\pi}{2^{k+1}}, \frac{\pi}{2^{k}}\right)$, they give us desired symmetric wavelet sets.

2. Cover $[2 k \pi, 2 k \pi+\pi), k \in \mathbf{N}$.

Fix $k \in \mathbf{N}$. There exists an integer $n_{k}$, such that $2^{n_{k}}[2 k \pi, 2 k \pi+\pi) \subset\left[\frac{\pi}{2}, \pi\right)$. Let $G_{k, 2}=\left[2 k \pi, 2 k \pi+\frac{\pi}{2}\right) \cup F_{k, 2}$, where $F_{k, 2}$ is $2 \pi$-translation congruent to $\left[\frac{\pi}{2}, \pi\right)$ and 2-dilation congruent to $\left[\frac{\pi}{2}, \pi\right) \backslash 2^{n_{k}}\left[2 k \pi, 2 k \pi+\frac{\pi}{2}\right)$. Similarly, let $G_{k, 3}=[2 k \pi+$ $\left.\frac{\pi}{2}, 2 k \pi+\pi\right) \cup F_{k, 3}$, where $F_{k, 3}$ is $2 \pi$-translation congruent to $\left[0, \frac{\pi}{2}\right)$ and 2-dilation congruent to $\left[\frac{\pi}{2}, \pi\right) \backslash 2^{n_{k}}\left[2 k \pi+\frac{\pi}{2}, 2 k \pi+\pi\right)$. The existence of $F_{k, 2}$ and $F_{k, 3}$ is due to Lemma 2.2. Since each of the sets $G_{k, 2}$ and $G_{k, 3}$ is $2 \pi$-translation congruent to $[0, \pi)$ and 2-dilation congruent to $\left[\frac{\pi}{2}, \pi\right)$, they give rise to symmetric wavelet sets.

3. Cover $[2 k \pi-\pi, 2 k \pi), k \in \mathbf{N}$.

If $k=1$, the positive part of the Shannon wavelet set will cover $[\pi, 2 \pi)$. Fix an integer $k>1$. There exists an integer $m_{k}$, such that $2^{m_{k}}[2 k \pi-\pi, 2 k \pi) \subset$ $\left[\frac{\pi}{4}, \frac{\pi}{2}\right)$. Let $G_{k, 4}=\left[2 k \pi-\pi, 2 k \pi-\frac{\pi}{2}\right) \cup F_{k, 4}$, where $F_{k, 4}$ is $2 \pi$-translation congruent to $\left[\frac{\pi}{4}, \frac{\pi}{2}\right) \cup\left[\frac{7}{4} \pi, 2 \pi\right)$ and 2-dilation congruent to $\left[\frac{\pi}{4}, \frac{\pi}{2}\right) \backslash 2^{m_{k}}[2 k \pi-\pi, 2 k \pi)$. The existence of $F_{k, 4}$ is due to Lemma 2.2. The sets $G_{k, 4}$ are $2 \pi$-translation congruent to $\left[\frac{\pi}{4}, \frac{\pi}{2}\right) \cup\left[\pi, \frac{3}{2} \pi\right) \cup\left[\frac{7}{4} \pi, 2 \pi\right)$ and 2-dilation congruent to $\left[\frac{\pi}{4}, \frac{\pi}{2}\right)$; so they give us symmetric wavelet sets.

Let $G_{k, 5}=\left[2 k \pi-\frac{\pi}{2}, 2 k \pi\right) \cup F_{k, 5}$, where $F_{k, 5}$ is $2 \pi$-translation congruent to $\left[\frac{\pi}{2}, \pi\right)$ and 2-dilation congruent to $\left[\frac{\pi}{2}, \pi\right) \backslash 2^{m_{k}+1}[2 k \pi-\pi, 2 k \pi)$. The existence of $F_{k, 5}$ is due to Lemma 2.2. The sets $G_{k, 5}$ are $2 \pi$-translation congruent to $\left[\frac{\pi}{2}, \pi\right) \cup\left[\frac{3}{2} \pi, 2 \pi\right)$ and 2-dilation congruent to $\left[\frac{\pi}{2}, \pi\right)$; so they give us symmetric wavelet sets.

That wavelet sets (not necessarily symmetric) cover the real line was first proven by Speegle (see Lemma 2.1). This result (or equivalently Proposition 2.3) implies that the set of all linear combinations of functions in $\widehat{\mathcal{W}}$ is dense in $L^{2}(\mathbb{R}, d \xi)$ and $L^{2}\left(\mathbb{R}, \frac{d \xi}{|\xi|}\right)$. Indeed, if $W$ is a wavelet set, then the family of functions $e_{n}(\xi)=$ $e^{i n \xi} \chi_{W}, n \in \mathbb{Z}$ forms an orthogonal basis of $L^{2}(W, d x)$. Since every $e_{n}$ is a Fourier transform of a wavelet and wavelet sets cover the real line, our claim follows.

The last fact we need for proving our main result is a characterization of operators unitary in $L^{2}(\mathbb{R}, d \xi)$ and $L^{2}\left(\mathbb{R}, \frac{d \xi}{|\xi|}\right)$. It is not hard to establish that if $T$ is a transformation unitary in $L^{2}\left(\mathbf{R}^{+}, d \xi\right)$ and $L^{2}\left(\mathbf{R}^{+}, \frac{d \xi}{|\xi|}\right)$, then $T$ is a unimodular multiplier, that is, $T f=u f$, where $|u|=1$ a.e. The matter is slightly more complicated in the case of $\mathbf{R}$ because of the symmetry of the real line. In order to deal with this case we introduce the notation $f^{+}=f \chi_{\mathbb{R}^{+}}, f^{-}=f \chi_{\mathbb{R}^{-}}$and $\widetilde{f}(\xi)=f(-\xi)$, where $f$ is a complex-valued function defined on $\mathbb{R}$. 
Proposition 2.4. If $T$ is unitary in $L^{2}(\mathbb{R}, d \xi)$ and $L^{2}\left(\mathbb{R}, \frac{d \xi}{|\xi|}\right)$, then for $f \in$ $L^{2}(\mathbb{R}, d \xi)$,

$$
T f=\left(f^{+}+\widetilde{f^{+}}\right) u+\left(f^{-}+\widetilde{f^{-}}\right) w
$$

where $u, w$ are complex-valued functions defined on $\mathbb{R}$ such that the matrix

$$
\left[\begin{array}{cc}
u^{+} & w^{+} \\
\widetilde{u^{-}} & \widetilde{w^{-}}
\end{array}\right]
$$

is unitary almost everywhere on $\mathbb{R}^{+}$.

Proof. We will denote the inner products in $L^{2}(\mathbb{R}, d \xi)$ and $L^{2}\left(\mathbb{R}, \frac{d \xi}{|\xi|}\right)$ by $\langle\cdot, \cdot\rangle_{d \xi}$ and $\langle\cdot, \cdot\rangle_{\frac{d \xi}{|\xi|}}$ respectively. Let $E=[-d,-c] \cup[a, b]$, where $0<a \leq b<\infty, 0<c \leq d<\infty$ and $h_{n}(\xi)=|\xi|^{n} \chi_{E}(\xi)$ for $n \in \mathbb{N} \cup\{0\}$. Of course we have $h_{n} \in L^{2}(\mathbb{R}, d \xi) \cap$ $L^{2}\left(\mathbb{R}, \frac{d \xi}{|\xi|}\right)$. So for every function $g \in L^{2}(\mathbb{R}, d \xi) \cap L^{2}\left(\mathbb{R}, \frac{d \xi}{|\xi|}\right)$ we can write

$$
\left\langle T\left(h_{n+1}\right), T g\right\rangle_{\frac{d \xi}{|\xi|}}=\left\langle h_{n+1}, g\right\rangle_{\frac{d \xi}{|\xi|}}=\left\langle h_{n}, g\right\rangle_{d \xi}=\left\langle T\left(h_{n}\right), T g\right\rangle_{d \xi},
$$

or simply

$$
\int_{\mathbf{R}} T\left(h_{n+1}\right) \overline{T g} \frac{d \xi}{|\xi|}=\int_{\mathbf{R}} T\left(h_{n}\right) \overline{T g} d \xi .
$$

Unitarity of $T$ assures that $\overline{T g}$ is an arbitrary function from $L^{2}(\mathbb{R}, d \xi) \cap L^{2}\left(\mathbb{R}, \frac{d \xi}{|\xi|}\right)$. Since the set $L^{2}(\mathbb{R}, d \xi) \cap L^{2}\left(\mathbb{R}, \frac{d \xi}{|\xi|}\right)$ is dense in $L^{2}(\mathbb{R}, d \xi)$, we have

$$
T\left(h_{n+1}\right)(\xi)=|\xi| T\left(h_{n}\right)(\xi) \quad \text { a.e. }
$$

Hence for every polynomial $p$,

$$
T\left(p^{\prime} \chi_{E}\right)=p^{\prime} T\left(\chi_{E}\right)
$$

where $p^{\prime}(\xi)=p(|\xi|)$.

This implies that for every even function $f \in L^{2}(\mathbb{R}, d \xi)$,

$$
T\left(f \chi_{E}\right)=f T\left(\chi_{E}\right) .
$$

Indeed, let $E_{n}=\left[-n,-\frac{1}{n}\right] \cup\left[\frac{1}{n}, n\right]$, for $n \in \mathbb{N}$ and fix an $n$ such that $E \subset E_{n}$. Then, by $(2.3)$ we have

$$
\chi_{E_{n}} T\left(p^{\prime} \chi_{E_{n}} \chi_{E}\right)=p^{\prime} \chi_{E_{n}} T\left(\chi_{E}\right) .
$$

Now let us consider an even function $f \in L^{2}(\mathbb{R}, d \xi)$ with $\operatorname{supp} f \subset E_{m}(m \leq n)$. We know that such a function can be approximated by functions of the form $p^{\prime} \chi_{E_{n}}$. Therefore, for every such function $f$ we obtain

$$
\chi_{E_{n}} T\left(f \chi_{E}\right)=f T\left(\chi_{E}\right) .
$$

Finally, since $\bigcup_{n=1}^{\infty} E_{n}$ covers $\mathbf{R}$, we conclude that

$$
T\left(f \chi_{E}\right)=f T\left(\chi_{E}\right),
$$

for any even function $f$ with supp $f \subset E_{m}$. The fact that $m$ is arbitrary implies that the above equation holds for all even $f \in L^{2}(\mathbb{R}, d \xi)$.

We will now try to determine $T f$ for an arbitrary $f \in L^{2}(\mathbb{R}, d \xi)$. Let $E_{n}{ }^{+}=$ $\left[\frac{1}{n}, n\right]$ and $E_{n}{ }^{-}=\left[-n,-\frac{1}{n}\right]$. By (2.4),

$$
\begin{aligned}
T\left(f \chi_{E_{n}}\right) & =T\left(\left(f^{+}+\widetilde{f^{+}}\right) \chi_{E_{n}+}\right)+T\left(\left(f^{-}+\widetilde{f^{-}}\right) \chi_{E_{n}-}\right) \\
& =\left(f^{+}+\widetilde{f^{+}}\right) T\left(\chi_{E_{n}}\right)+\left(f^{-}+\widetilde{f^{-}}\right) T\left(\chi_{E_{n}}\right) .
\end{aligned}
$$


We claim that

$$
T\left(\chi_{E_{n}+}\right)=u \chi_{E_{n}}
$$

for some complex-valued function $u$ defined on $\mathbb{R}$ that does not depend on $n \in \mathbb{N}$. To prove the claim let us define the function $u$ as follows:

$$
\left.u\right|_{E_{n}}=\left.T\left(\chi_{E_{n}+}\right)\right|_{E_{n}} .
$$

To see that the definition is correct it is enough to check that for $n \leq m$,

$$
\left.\left(\left.u\right|_{E_{m}}\right)\right|_{E_{n}}=\left.u\right|_{E_{n}},
$$

which holds since by (2.4) we have

$$
\left.\left(\left.T\left(\chi_{E_{m}^{+}}\right)\right|_{E_{m}}\right)\right|_{E_{n}}=\left.T\left(\chi_{E_{m}^{+}}\right)\right|_{E_{n}}=\left.\left(\chi_{E_{n}} T\left(\chi_{E_{m}^{+}}\right)\right)\right|_{E_{n}}=\left.T\left(\chi_{E_{n}^{+}}\right)\right|_{E_{n}} .
$$

Moreover, by (2.4), $u$ satisfies

$$
T\left(\chi_{E_{n}^{+}}\right)=T\left(\chi_{E_{n}} \chi_{E_{n}^{+}}\right)=\chi_{E_{n}} T\left(\chi_{E_{n}^{+}}\right)=u \chi_{E_{n}},
$$

proving our claim.

A similar argument leads to

$$
T\left(\chi_{E_{n}-}\right)=w \chi_{E_{n}}
$$

for another complex-valued function $w$ defined on $\mathbb{R}$. Therefore, formula (2.5) becomes

$$
T\left(f \chi_{E_{n}}\right)=\left(f^{+}+\widetilde{f^{+}}\right) u \chi_{E_{n}}+\left(f^{-}+\widetilde{f^{-}}\right) w \chi_{E_{n}} .
$$

Since the left-hand side of the above equality converges to $T f$ in $L^{2}(\mathbb{R}, d \xi)$ and the right-hand side converges pointwise to $\left(f^{+}+\widetilde{f^{+}}\right) u+\left(f^{-}+\widetilde{f^{-}}\right) w$, we obtain the desired formula for $T f$.

The last step of our proof is to show that the matrix

$$
\left[\begin{array}{cc}
u^{+} & w^{+} \\
\widetilde{u^{-}} & w^{-}
\end{array}\right]
$$

is unitary almost everywhere on $\mathbb{R}^{+}$. To do so let us notice that for every function $f \in L^{2}(\mathbb{R}, d \xi)$ such that $f^{-}=0$ we have

$$
T f=\left(f^{+}+\widetilde{f^{+}}\right) u .
$$

Thus, using the unitarity of $T$ on $L^{2}(\mathbb{R}, d \xi)$ we obtain

$$
\int_{\mathbb{R}^{+}}\left|f^{+}\right|^{2} d x=\int_{\mathbb{R}^{+}}\left|f^{+}\right|^{2}\left|u^{+}\right|^{2} d x+\int_{\mathbb{R}^{-}}\left|\widetilde{f^{+}}\right|^{2}\left|u^{-}\right|^{2} d x=\int_{\mathbb{R}^{+}}\left|f^{+}\right|^{2}\left(\left|u^{+}\right|^{2}+\left|\widetilde{u^{-}}\right|^{2}\right) d x,
$$

which implies that on $\mathbb{R}^{+}$,

$$
\left|u^{+}\right|^{2}+\left|\widetilde{u^{-}}\right|^{2}=1
$$

Similarly, by assuming $f^{+}=0$, we obtain

$$
\left|w^{+}\right|^{2}+\left|\widetilde{w^{-}}\right|^{2}=1
$$

almost everywhere on $\mathbb{R}^{+}$. To prove the third relationship that we need, let us note that again by the unitarity of $T$,

$$
\begin{aligned}
\int_{\mathbb{R}}|f|^{2} d x & =\int_{\mathbb{R}^{+}}\left|f^{+} u^{+}+\widetilde{f^{-}} w^{+}\right|^{2} d x+\int_{\mathbb{R}^{-}}\left|f^{-} w^{-}+\widetilde{f^{+}} u^{-}\right|^{2} d x \\
& =\int_{\mathbb{R}}|f|^{2} d x+\int_{\mathbb{R}^{+}} 4 \operatorname{Re}\left(f^{+} \overline{\widetilde{f-}}\left(u^{+} \overline{w^{+}}+\widetilde{u^{-}} \widetilde{w^{-}}\right)\right) d x
\end{aligned}
$$


which implies

$$
\int_{\mathbb{R}^{+}} \operatorname{Re}\left(f^{+} \overline{\widetilde{f-}}\left(u^{+} \overline{w^{+}}+\widetilde{u^{-}} \overline{\widetilde{w^{-}}}\right)\right) d x=0
$$

Now by considering two special cases $\widetilde{f^{-}}=f^{+}$and $\widetilde{f^{-}}=i f^{+}$we obtain

$$
\int_{\mathbb{R}^{+}}\left|f^{+}\right|^{2}\left(u^{+} \overline{w^{+}}+\widetilde{u^{-}} \overline{\widetilde{w^{-}}}\right) d x=0
$$

Therefore, $u^{+} \overline{w^{+}}+\widetilde{u^{-}} \widetilde{\widetilde{w^{-}}}=0$ almost everywhere on $\mathbb{R}^{+}$.

\section{MAIN RESUlT}

The only known continuous linear operators that preserve wavelets are given by multiplication by wavelet multipliers and the reflection in the argument. These transformations are unitary in $L^{2}(\mathbb{R}, d \xi)$ and $L^{2}\left(\mathbb{R}, \frac{d \xi}{|\xi|}\right)$. Our main result is the following characterization.

Theorem 3.1. Every operator $T$ that satisfies $T(\widehat{\mathcal{W}}) \subset \widehat{\mathcal{W}}$ and is unitary in $L^{2}(\mathbb{R}, d \xi)$ and $L^{2}\left(\mathbb{R}, \frac{d \xi}{|\xi|}\right)$ is of the form

$$
T f(\xi)=v(\xi) f(I(\xi)), \quad f \in L^{2}(\mathbb{R}, d \xi),
$$

where $v$ is a wavelet multiplier and $I= \pm i d$.

Proof. By Proposition 2.4 the operator $T$ is given by

$$
T f=\left(f^{+}+\widetilde{f^{+}}\right) u+\left(f^{-}+\widetilde{f^{-}}\right) w,
$$

where the matrix

$$
M=\left[\begin{array}{cc}
u^{+} & w^{+} \\
\widetilde{u^{-}} & w^{-}
\end{array}\right]
$$

is unitary almost everywhere on $\mathbb{R}^{+}$.

Using symmetric wavelet sets we will show that the functions $u, w$ are characteristic functions of some sets, which will imply that

$$
T f(\xi)=v(\xi) f\left(I_{A}(\xi)\right)
$$

where $v$ is a unimodular function, $A$ is a symmetric subset of $\mathbb{R}$ and

$$
I_{A}(\xi)=\left\{\begin{array}{rr}
-\xi & \text { for } \xi \in A, \\
\xi & \text { for } \xi \notin A .
\end{array}\right.
$$

After that, using non-symmetric wavelet sets we will show that $I_{A}$ can preserve wavelet sets if and only if it is invariant under translations and dilations, that is, $A+2 \pi=A$, and $2 A=A$, which implies that $A$ is either $\mathbb{R}$ or $\emptyset$.

Let us fix a symmetric wavelet set $W$. If $f$ is a function such that $|f|=\chi_{W}$, then $T f$ is a Fourier transform of some wavelet $\eta$ (see the comment after (2.2)). Note that the special form of $T$ implies that $\operatorname{supp} T f \subset W$, which forces $|\hat{\eta}|=\chi_{W}$, that is, $|T f|=\chi_{W}$. Let us define $W^{+}$as $W \cap \mathbb{R}^{+}$and $W^{-}$as $W \cap \mathbb{R}^{-}$. On $W^{+}$we have

$$
1=|T f|=\left|f^{+} u^{+}+\widetilde{f^{-}} w^{+}\right|^{2}=\left|u^{+}\right|^{2}+\left|w^{+}\right|^{2}+2 \operatorname{Re}\left(f+\widetilde{\widetilde{f-}} u^{+} \overline{w^{+}}\right) .
$$

Since $\left|u^{+}\right|^{2}+\left|w^{+}\right|^{2}=1$, therefore

$$
\operatorname{Re}\left(f^{+} \overline{\widetilde{f-}} u^{+} \overline{w^{+}}\right)=0
$$


a.e. on $W^{+}$. By considering two special kinds of functions $f$ with $\widetilde{f^{-}}=f^{+}$and $\widetilde{f^{-}}=i f^{+}$we obtain $u^{+} \overline{w^{+}}=0$ a.e. on $W^{+}$. This means that there is a subset of $W^{+}$, call it $A_{W^{+}}$, such that $\left|u^{+}\right|=0,\left|w^{+}\right|=1$ on $A_{W^{+}}$and $\left|u^{+}\right|=1,\left|w^{+}\right|=0$ on $W^{+} \backslash A_{W^{+}}$. Moreover, using $\left|u^{+}\right|^{2}+\left|\widetilde{u^{-}}\right|^{2}=1$ and $\left|w^{+}\right|^{2}+\left|\widetilde{w^{-}}\right|^{2}=1$, we conclude that $\left|u^{-}\right|=1,\left|w^{-}\right|=0$ on $-A_{W^{+}}$and $\left|u^{-}\right|=0,\left|w^{-}\right|=1$ on $W^{-} \backslash-A_{W^{+}}$.

Since symmetric wavelet sets cover the real line, we obtain a global symmetric set $A \subset \mathbf{R}$ such that $\left|u^{+}\right|=0,\left|w^{+}\right|=1$ on $A^{+}=A \cap \mathbf{R}^{+},\left|u^{+}\right|=1,\left|w^{+}\right|=0$ on $\mathbb{R}^{+} \backslash A^{+}$and $\left|u^{-}\right|=1,\left|w^{-}\right|=0$ on $A^{-}=A \cap \mathbf{R}^{-},\left|u^{-}\right|=0,\left|w^{-}\right|=1$ on $\mathbb{R}^{-} \backslash A^{-}$.

To finish the proof of the first step, let us define a function $v$ as follows:

$$
\begin{aligned}
\left.v\right|_{A^{+}} & =\left.w^{+}\right|_{A^{+}}, \\
\left.v\right|_{\mathbb{R}^{+} \backslash A^{+}} & =\left.u^{+}\right|_{\mathbb{R}^{+} \backslash A^{+}}, \\
\left.v\right|_{A^{-}} & =\left.u^{-}\right|_{A^{-}}, \\
\left.v\right|_{\mathbb{R}^{-} \backslash A^{-}} & =\left.w^{-}\right|_{\mathbb{R}^{-} \backslash A^{-}} .
\end{aligned}
$$

It is easy to check that with this definition the operator $T$ can be described as

$$
T f(\xi)=v(\xi) f\left(I_{A}(\xi)\right)
$$

where

$$
I_{A}(\xi)=\left\{\begin{array}{rc}
-\xi & \text { for } \xi \in A, \\
\xi & \text { for } \xi \notin A .
\end{array}\right.
$$

From (3.1) it follows that if $W$ is a wavelet set, then $\left|T\left(\chi_{W}\right)\right|=\chi_{W^{\prime}}$, where $W^{\prime}$ is also a wavelet set. We are going to prove that in order to preserve equation (2.1), $A$ has to satisfy $A+2 \pi=A$ and, similarly, to preserve equation (2.2), we must have $2 A=A$.

To show that $A+2 \pi \subset A$ a.e., it suffices to show that almost every point $\xi_{0} \in A$ has an open neighborhood $U$ such that $\xi+2 \pi \in A$ for almost all $\xi \in U \cap A$. In fact, let $\xi_{0} \in A$ be a point such that $\xi_{0} \neq k \pi$ for any integer $k$. Then by Lemma 2.1 there is a wavelet set $W$ and an $\varepsilon>0$ such that the sets $U=\left[\xi_{0}-\varepsilon, \xi_{0}+\varepsilon\right]$ and $U^{\prime}=-U-2 \pi$ are contained in $W$. By (2.1) we obtain that for almost all $\xi \in U$,

$$
\begin{gathered}
\sum_{k \in \mathbb{Z}}\left|\chi_{W}(\xi+2 k \pi)\right|=1, \\
\sum_{k \in \mathbb{Z}}\left|\chi_{W}(-\xi+2 k \pi)\right|=1, \\
\sum_{k \in \mathbb{Z}}\left|T\left(\chi_{W}\right)(\xi+2 k \pi)\right|=1 .
\end{gathered}
$$

Let us fix a $\xi \in U \cap A$ for which the above equations are true. By (3.4) there is a unique $k_{0}$ such that

$$
\left|T\left(\chi_{W}\right)\left(\xi+2 k_{0} \pi\right)\right|=1 .
$$

We claim that $\xi+2 k_{0} \pi \in A$. Indeed, if not, then by (3.1),

$$
1=\left|T\left(\chi_{W}\right)\left(\xi+2 k_{0} \pi\right)\right|=\left|\chi_{W}\left(\xi+2 k_{0} \pi\right)\right| .
$$

So by (3.2) we obtain $k_{0}=0$ (since $\xi \in W$ ), but because we assumed $\xi+2 k_{0} \pi \notin A$, this is a contradiction with $\xi \in A$. Hence $\xi+2 k_{0} \pi \in A$, and it remains to prove that $k_{0}=1$. By (3.1) again,

$$
1=\left|T\left(\chi_{W}\right)\left(\xi+2 k_{0} \pi\right)\right|=\left|\chi_{W}\left(-\xi-2 k_{0} \pi\right)\right|,
$$


which together with (3.3) implies that $k_{0}=1$ (since $-\xi-2 \pi \in U^{\prime} \subset W$ ); thus $\xi+2 \pi \in A$.

To prove the other inclusion, $A+2 \pi \supset A$, we apply Lemma 2.1 to find a wavelet set that contains $\xi_{0}$ and $-\xi_{0}+2 \pi$ (instead of $\xi_{0}$ and $-\xi_{0}-2 \pi$ ) and proceed as in the previous case.

Similarly, using (2.2) we prove that $2 A \subset A$. By Lemma 2.1 for each $\xi_{0} \neq q \pi$, $q \in \mathbb{Q}$ we can find a wavelet set $W$ containing $\xi_{0}$ and $-2 \xi_{0}$. After fixing a $\xi \in U \cap A$, where $\xi_{0} \in U \subset W$ and $U$ open, we get again that for some $j_{0}$,

$$
\left|T\left(\chi_{W}\right)\left(2^{j_{0}} \xi\right)\right|=1 .
$$

For the same reasons as in the previous case we must have $2^{j_{0}} \xi \in A$ and $j_{0}=1$. Considering a wavelet set containing $\xi_{0}$ and $-\frac{1}{2} \xi_{0}$, we obtain the other inclusion, $\frac{1}{2} A \subset A$, which proves that $2 A=A$.

To end our proof we observe that $\chi_{A}$ is $2^{-n} \pi$-periodic for every $n \in \mathbf{N}$. Thus, using the Haar orthonormal basis of $L^{2}([0,2 \pi])$, we establish that $\chi_{A}$ is constant on $[0,2 \pi]$, hence also on $\mathbf{R}$. Therefore, either $A=\emptyset$ or $A=\mathbf{R}$.

\section{REMARKS}

As we mentioned before it is natural to ask whether all continuous linear transformations $T$ of $L^{2}(\mathbb{R}, d \xi)$ preserving $\widehat{\mathcal{W}}$ must be unitary. The main reason for this is that such operators preserve the norm of elements of $\widehat{\mathcal{W}}$. The same question can be stated for the $L^{2}\left(\mathbb{R}, \frac{d \xi}{|\xi|}\right)$-norm as well.

It is not hard to see that if $E$ and $F$ are disjoint subsets of a wavelet set $W$, then $T$ preserves the orthogonality (in both norms) of the characteristic functions of $E$ and $F$, provided that $T$ preserves $\widehat{\mathcal{W}}$ (it is enough to consider $\| T\left(a_{1} \chi_{E}+a_{2} \chi_{F}+\right.$ $\left.a_{3} \chi_{W \backslash(E \cup F)}\right) \|$ with appropriate choices of $\left.a_{1}, a_{2}, a_{3} \in\{1,-1, i,-i\}\right)$. Therefore we can define a measure $d \mu$ on subsets of wavelet sets by setting $\int_{\mathbf{R}} \chi_{E} d \mu=\left\|T \chi_{E}\right\|^{2}$. Since for every point on $\mathbf{R}$ (except the origin) there is a neighborhood of it that is contained in a wavelet set, the measure can be extended to $\mathbf{R}$. Proving that this measure is equal to $d \xi$ in the $L^{2}(\mathbb{R}, d \xi)$ case would show that $T$ is an isometry of $L^{2}(\mathbb{R}, d \xi)$. Similarly, the equality $d \mu=\frac{d \xi}{|\xi|}$ in the $L^{2}\left(\mathbb{R}, \frac{d \xi}{|\xi|}\right)$ case would imply that $T$ is an isometry of $L^{2}\left(\mathbb{R}, \frac{d \xi}{|\xi|}\right)$.

\section{ACKNOWLEDGEMENTS}

We thank David Larson for communicating this problem to us and the referee for improving our paper. The first author acknowledges helpful discussions with Guido Weiss.

\section{REFERENCES}

[B] Bownik, M., On characterizations of multiwavelets in $L^{2}\left(\mathbb{R}^{n}\right)$, Proc. Amer. Math. Soc. 129 (2001), 3265-3274. MR 2002g:42046

[GS] Garrigós, G. and Speegle, D., Completeness in the set of wavelets, Proc. Amer. Math. Soc. 128 (2000), 1157-1166. MR 2000i:42021

[HKLS] Ha, Y., Kang, H., Lee, J., and Seo, J., Unimodular wavelets for $L^{2}$ and the Hardy space $H^{2}$, Michigan Math. J. 41 (1994), 345-361. MR 95g:42050

[HW] Hernández, E. and Weiss, G., A first course on wavelets, Studies in Advanced Mathematics, CRC Press, Boca Raton, FL, 1996. MR 97i:42015 
[L] Larson, D. R., Von Neumann algebras and wavelets, Operator algebras and applications (Samos, 1996), 267-312, NATO Adv. Sci. Inst. Ser. C Math. Phys. Sci., 495, Kluwer Acad. Publ., Dordrecht, 1997. MR 98g:46091

[R] Rzeszotnik, Z., Calderón's condition and wavelets, Collect. Math. 52 (2001), 181-191. MR 2002j:42039

[S] Speegle, D. M., The s-elementary wavelets are path-connected, Proc. Amer. Math. Soc. 127 (1999), 223-233. MR 99b:42045

[W] Weiss, G., personal communication.

[WUTAM] The Wutam Consortium, Basic properties of wavelets, J. Fourier Anal. Appl. 4 (1998), 575-594. MR 99i:42056

[Z] Zhang, X., Wavelet sets and frame sets, Ph. D. Thesis, Texas A\&M University, 2002.

Institute of Mathematics, University of Wroclaw, Wroclaw, Poland

Department of Mathematics, University of Texas at Austin, Austin, Texas 78712

E-mail address: zioma@math.utexas.edu

Department of Mathematics, Texas A\&M University, College Station, Texas 77843 